\title{
TECNICHE PER MASCHERARE IL SAPORE AMARO DEI PRODOTTI FARMACEUTICI
}

\author{
ELISABETTA BRENNA (*) \\ Nota presentata dal m.e. C. Fuganti \\ (Adunanza del 23 marzo 2017)
}

SuNTO. - Nel testo vengono illustrate le principali tecniche usate in campo farmaceutico per mascherare il sapore amaro delle specialità medicinali contenute e rendere il prodotto più gradevole per l'assunzione orale da parte del paziente.

$* * *$

ABSTRACT. - The text describes the main techniques used in the pharmaceutical field to mask the bitter taste of active pharmaceutical ingredients and make the product more pleasant for oral intake by the patient.

\section{INTRODUZIONE}

Il sapore gioca un ruolo fattore fondamentale nel determinare la gradevolezza delle formulazioni farmaceutiche per uso orale, così da ottenere l'apprezzamento da parte del paziente. Il sapore conferisce al farmaco una caratteristica specifica ed unica, che lo può rendere preferibile ad altri prodotti. Questo aspetto è di particolare importanza nell'ambito dei medicinali da banco e di quelli per uso pediatrico.

La sensazione di amaro provocata in bocca da molti principi attivi farmaceutici è uno degli ostacoli maggiori che si incontrano nella messa

(*) Dipartimento di Chimica, Materiali ed Ingegneria Chimica “G. Natta", Politecnico di Milano, Italy. E-mail: mariaelisabetta.brenna@polimi.it 
a punto di sistemi che consentano la somministrazione del farmaco per via orale (soluzioni, sospensioni, pastiglie/lastrine da sciogliere in bocca, gomme da masticare), modalità che consente maggior praticità di ingestione, oltre che rapidità di azione e di assorbimento. Le alternative possibili per i farmaci amari sono la somministrazione endovenosa, che è più laboriosa ed invasiva anche se più efficace, o mediante compresse rivestite, che però può presentare un problema per bambini e anziani, o per pazienti con particolari patologie. La legislazione europea corrente ha imposto un piano di sviluppo preciso per il controllo dei sapori amari e sgradevoli nei farmaci ad uso pediatrico. Il cattivo sapore di una medicina, infatti, può addirittura pregiudicarne l'assunzione e mettere a rischio i benefici ad essa correlati. D'altro canto, una medicina non deve essere "troppo buona", per evitare abusi ed assuefazioni.

Le tecniche per mascherare il sapore amaro dei medicinali si basano su tre approcci fondamentali: i) aggiunta di dolcificanti, aromi ed agenti effervescenti che coprano il sapore sgradevole; ii) sequestro della sostanza amara con metodi fisici, per evitare che essa venga in contatto con i calici gustativi; iii) utilizzo di composti che interagiscono con i recettori gustativi bloccandoli.

\section{Aggiunta di DOLCIFICANTI, AROMi ED ALTRI ECCIPIENTI}

L'aggiunta di dolcificanti ed aromi è il metodo più semplice per coprire il sapore amaro di un farmaco.

I dolcificanti sono di solito molto solubili in acqua, si sciolgono nella saliva e coprono i calici gustativi presenti sulla lingua, ritardando l'interazione con il farmaco amaro. Gli aromi conferiscono alla preparazione un sapore netto che tende a prevalere e sono usati in combinazione con altri agenti primari. Certi aromi raffrescanti, come il mentolo, "stordiscono" i calici gustativi e ritardano la percezione dell'amaro.

Anche lo zinco solfato si è rivelato efficace [1] nell'inibire il sapore amaro di alcune sostanze, come il chinico cloridrato, il tetralone e il benzoato di denatonio. Non ha invece effetto sul saccarosio ottaacetato, sulla pseudoefedrina e sul destrometorfano. La sua utilità è compromessa dal fatto che è anche un buon inibitore della dolcezza e potrebbe interferire in generale con il sapore di formulazioni complesse. L'unico dolcificante che non viene inibito dallo zinco solfato è il sodio ciclamato. 
Il sapore sgradevole di certi colluttori e gocce per la tosse, contenenti medicinali e altre sostanze amare come l'olio di eucaliptolo, possono essere coperti usando fencone, borneolo e isoborneolo.[2] In alcuni dentifrici, il benzetonio cloruro viene mascherato con estratti di stevia con proprietà dolcificanti.[3] L'aggiunta di agenti anestetizzanti come il sodio fenolato a fili interdentali medicati con aspirina ha la funzione di stordire i calici gustativi per 4-5 secondi, rendendo impercettibile il sapore amaro dell'aspirina.[4] L'olio essenziale di chiodi di garofano, ad esempio, viene usato per mascherare il sapore amaro di analgesici, espettoranti, antitussivi, decongestionanti, grazie alla combinazione del suo sapore speziato e di un leggero effetto anestetico. Per sostenerne le capacità coprenti è possibile aggiungere aroma miele $\mathrm{o}$ vaniglia.

La combinazione di certi aromi con l'effetto di effervescenza, prodotto da acido citrico e sodio bicarbonato, è utile per coprire il sapore amaro di alcuni prodotti per i quali sia prevista la dissoluzione in acqua solo all'atto della somministrazione.

L'aspartame (Fig. 1) è il dolcificante più usato per ridurre il sapore amaro. Una concentrazione pari allo $0.8 \%$ è sufficiente per ridurre del $25 \%$ la sensazione di amaro prodotta dal paracetamolo. Altri dolcificanti artificiali, come la neoesperidina diidrocalcone (Fig. 1) ed il corrispondente glucoside, sono in grado di mascherare amaro e salato grazie alla loro dolcezza duratura. La loro efficacia viene dal fatto che il profilo di gusto di una sostanza amara appare più lentamente nel tempo rispetto alla normale durata di un dolcificante.[5]

A prodotti farmaceutici contenenti saponine con proprietà anticolesterolemiche vengono aggiunti amminoacidi, quali la glicina e la alanina, per il controllo del sapore amaro.<smiles>COC(=O)C(Cc1ccccc1)NC(=O)C(N)CC(=O)O</smiles>

aspartame<smiles>COc1ccc(CCC(=O)c2c(O)cc(OC3OC(CO)[C@@H](O)[C@H](O)[C@H]3OC3O[C@H](C)[C@@H](O)[C@H](O)[C@H]3O)cc2O)cc1O</smiles>

neoesperidina diidrocalcone

Fig. 1 - Strutture dell'aspartame e della neoesperidina diidrocalcone. 


\section{FORMAZIONE DI COMPLESSI DI INCLUSIONE}

Tale approccio sfrutta la possibilità che la molecola di farmaco si adatti alla cavità di un agente complessante, a dare un complesso stabile. Il metodo ha efficacia nell'attenuare il sapore amaro, sia perché risulta diminuita la solubilità orale del farmaco, sia perché diminuisce la quantità di sostanza esposta ai calici gustativi. Gli agenti complessanti più usati sono le ciclodestrine a 6,7 oppure 8 unità glucopiranosiche collegate da legami $\alpha$-glicosidici $(\alpha, \beta$, e $\gamma$-ciclodestrine, rispettivamente) (Fig. 2).

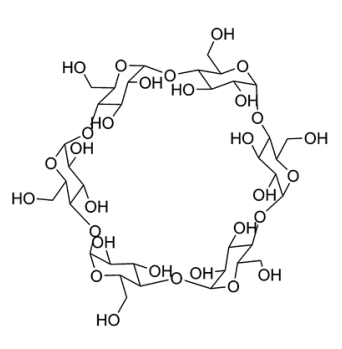

$\alpha$-ciclodestrina

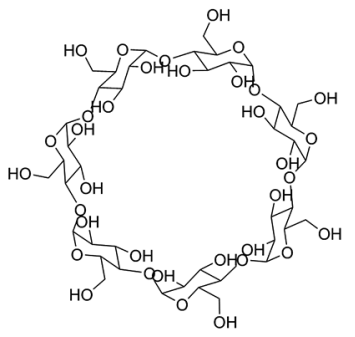

$\beta$-ciclodestrina

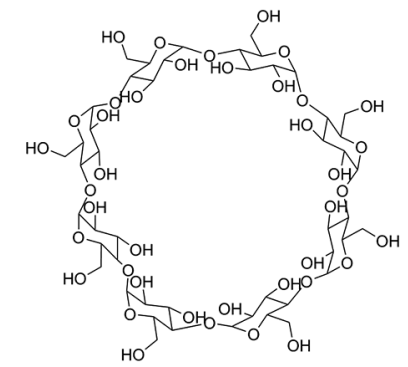

$\gamma$-ciclodestrina

Fig. 2 - Struttura delle più comuni ciclodestrine.

Le ciclodestrine danno luogo ad una caratteristica struttura a tronco di cono con un diametro interno di 5-10 $\AA$, una superficie interna idrofobica ed una esterna idrofilica. In soluzione il cono è idratato, ma piccole molecole idrofobiche o porzioni idrofobiche di molecole grandi possono legarsi mediante forze di Van der Waals alla cavità interna. L'affinità della ciclodestrina per una data sostanza dipende dal valore della costante di associazione $\left(\mathrm{K}_{\text {ass }}\right)$.

La velocità di formazione dei complessi si avvicina al limite di diffusione, cosicchè è possibile avere un buon eccesso di ciclodestrine presenti nel prodotto farmaceutico prima che sia assunto.

La cetrazina forma dei complessi 1:1 in soluzione con le ciclodestrine $\alpha \beta \varepsilon \gamma$.[6] Le ciclodestrine più piccole $\alpha$ e $\beta$ formano un complesso stabile con una parte della cetrazina, mentre la ciclodestrina più grande forma un complesso meno stabile con l'intera molecola. Le ciclodestrine $\alpha$ e $\beta$ sopprimono il sapore amaro della cetrazina, mentre la ciclodestrina $\gamma$ è inefficace, forse appunto per il basso valore di $K_{\text {ass }}$. E' stata messa a punto, ad esempio, una gomma da masticare contenente cetrazina e ciclodestrine.[7] Queste ultime favoriscono il rilascio del 
principio attivo dalla gomma durante la masticazione. Tuttavia, a causa della velocità di interazione, avere semplicemente le ciclodestrine nella formulazione ha gli stessi effetti che usare i complessi pre-formati.

In alcuni casi, un terzo agente viene aggiunto per promuovere l'interazione ospite-ciclodestrina. Per esempio, la famotidina ha una $\mathrm{K}_{\text {ass }}$ relativamente bassa con la $\beta$-ciclodestrina, ma l'aggiunta di idrossipropil metil cellulosa aumenta l'interazione di legame e diminuisce il sapore amaro.[8]

\section{FORMAZIONE DI RIVESTIMENTI CON MATERIALI POLIMERICI}

Il rivestimento è uno dei metodi più efficaci usato comunemente per coprire il sapore amaro. Polimeri idrofobici, idrofilici, e lipidi possono essere impiegati come materiali per il rivestimento, da soli o in combinazione, in soluzioni mono o multistrato, in processi di preparazione condotti in acqua o in solvente organico. Con questa tecnica si impedisce che il farmaco amaro venga in contatto con i recettori gustativi quando viene introdotto nella cavità orale. La scelta del materiale di rivestimento deve essere fatta, però, tenendo conto che non deve alterare il profilo di rilascio del farmaco in vivo. Agenti inerti di rivestimento che vengono usati sono: il polivinilpirrolidone, la gelatina, la metil e la etil cellulosa.

Ad esempio il sapore amaro dell'ibuprofen è stato coperto usando delle microcapsule, preparate con la tecnica dell' air suspension coating. Le microcapsule hanno un cuore di ibuprofen microcristallino, rivestito di copolimero acrilico e sono usate per creare un prodotto masticabile senza sapore amaro.

Uno dei metodi più efficienti per ottenere le microcapsule rivestite è l'impiego dei processori a letto fluido. Gli ingredienti sono miscelati e preriscaldati da un flusso di aria calda diretto verso l'alto. La granulazione ha luogo nebulizzando un liquido nella polvere fluidificata. Successivamente i granuli sono essiccati con aria calda. Una seconda alternativa è rappresentata dalla deposizione di strati successivi di un composto attivo su un nucleo di materiale inerte, ad esempio sfere di zucchero. Il farmaco amaro viene sciolto o disperso in un solvente, che può essere anche acquoso, insieme con un legante che consenta l'aderenza delle molecole di farmaco al substrato inerte. Come leganti vengono usati l'idrossipropilcellulosa (HPC), polivinilpirrolidone, carbossimetilcellulosa. Le particelle rivestite di strati di farmaco sono successivamente ricoperte con lo strato di polimero. Come polimeri si usano: 
etilcellulosa, Eudragit E-100, HPC, polivinilalcol e polivinilacetato.

Un terzo approccio consiste nell'effettuare la granulazione del farmaco e poi rivestirlo di polimero. La granulazione fa diminuire l'area superficiale delle particelle di farmaco, aumentandone le dimensioni. In tal modo diminuisce la quantità di polimero da usare. Per questi rivestimenti possono essere usate anche proteine, quali la zeina, la gliadina e l'ordeina o la gelatina.

\section{FormAZIONE DI COMPLESSI CON RESINE A SCAMBIO IONICO}

Le resine a scambio ionico sono polimeri ad alto peso molecolare con gruppi funzionali acidi o basici. Possono essere usate per legare i farmaci mediante esposizione ripetuta della resina alla soluzione contenente il farmaco in una colonna cromatografica, oppure mediante contatto prolungato della resina nella soluzione del farmaco. Le molecole di farmaco sono legate alla resina mediante interazioni ioniche, tali che la dissociazione non avvenga nelle condizioni di $\mathrm{pH}$ della saliva. Il rilascio del farmaco avviene più avanti nel tratto gastrointestinale. Le resine acide a scambio cationico sono usate con farmaci basici, quelle basiche a scambio anionico sono usate per farmaci acidi. Ad esempio, la formazione di un complesso chinolone-resina consente di eliminar il sapore amaro dei chinoloni e ottenere una formulazione liquida per uso orale.[9] Il chinolone viene sciolto in solvente acquoso, viene aggiunta la resina a dare il complesso. Questo viene poi sospeso direttamente in uno sciroppo (estratto di malto) con l'aggiunta di un agente anti-caking (silice colloidale) ed un conservante (acido sorbico).

\section{PREPARAZIONE DI PRO-FARMACI}

Per profarmaco si intende un precursore inerte di un farmaco che mediante una reazione che avvenga in vivo possa liberare la sostanza farmacologicamente attiva. Il sapore amaro di una sostanza deriva dall'efficienza con cui il recettore dell'amaro interagisce con la sostanza stessa, che dipende a sua volta dalla struttura chimica della sostanza. Modificando la struttura della sostanza possiamo modificarne la percezione gustativa. L'altra modalità di azione è la riduzione della solubilità nella saliva.

Gruppi carbossilici ed alcolici si prestano ad essere derivatizzati 
mediante esterificazione. Gli enzimi esterasi presenti poi nell'organismo possono rilasciare il principio attivo per idrolisi. Questo è l'approccio usato nel preparare l'estere palmitico della clindamicina (Fig. 3).

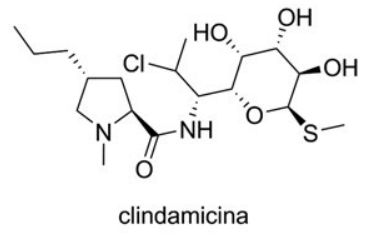

Fig. 3 - Struttura della clindamicina.

\section{USO DI BITTER RECEPTOR BLOCKERS}

Un approccio che può essere utilizzato per mascherare il sapore amaro è quello di utilizzare delle molecole che inibiscano l'attivazione dei recettori del gusto amaro presenti sulla nostra lingua.

Per 21 dei 25 recettori del gusto amaro sono stati identificati uno o più agonisti.[10] Sono invece assai rari i composti che sono stati trovati agire come antagonisti di tali recettori.[11]

Il composto più conosciuto e potente è l'acido 4-(2,2,3-trimetilciclopentil)butanoico (GIV3727, Fig. 4), preparato e studiato dalla Givaudan nel 2010: è in grado di attenuare il retrogusto amaro della saccarina e dell'acesulfame $\mathrm{K}$, inibendo l'attivazione dei recettori of hTAS2R31 and hTAS2R43.

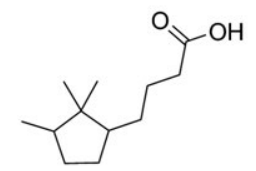

acido 4-(2,2,3-trimetilciclopentil)-butanoico (GIV3727)

Fig. 4 - Struttura del composto GIV3727.

Un altro composto in grado di diminuire l'attivazione dei recettori dell'amaro è l'acido $p$-(dipropilsulfamil)benzoico (noto come probenecid) (Fig. 5). Tale sostanza è in gardo di inibire l'attivazione dei recettori hTAS2R16, hTAS2R38, and hTAS2R43, e di sopprimere il sapore amaro della salicina nei test sensoriali. Esistono, invece, delle sostanze che si comportano come agonisti verso un certo set di recettori e come antago- 
nisti verso un altro set. Si tratta dei due lattoni sesquiterpenici $3 \beta$-idrossidiidrocostunolide (3HDC) and $3 \beta$-idrossipelenolide (3HP) (Fig. 5).<smiles>CCCN(CCC)S(=O)(=O)c1ccc(C(=O)O)cc1</smiles>

acido $p$-(dipropilsulfamil)benzoico<smiles>CC1=CC2=CC[C@@H](O)CC[C@H]2[C@@H](C)C(=O)O1</smiles>

3ß-idrossidiidrocostunolide

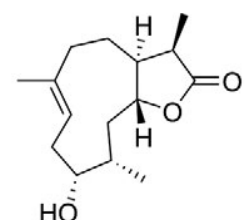

$3 \beta$-idrossipelenolide

Fig. 5 - Struttura di alcuni composti usati per ridurre l'attivazione dei recettori del sapore amaro.

Nel 2013 ricercatori della Unilever hanno riportato che tre flavanoni, 4'-fluoro-6- metossiflavanone, 6,3'-dimetossiflavanone, e 6metossiflavanone (Fig. 6), mostrano, in ordine di potenza decrescente, comportamento inibitorio verso l'attivazione del recettore hTAS2R39 ad opera della epigallocatechina gallato (ECG). Il 6-metossiflavanone inibisce anche l'attivazione del recettore hTAS2R14, sempre attivato dalla ECG. Lo stesso effetto è stato osservato con un agonista strutturalmente differente, il benzoato di denatonio.<smiles>COc1ccc2c(c1)C(=O)CC(c1ccc(F)cc1)O2</smiles>

4'-fluoro-6- metossiflavanone<smiles>COc1cccc(C2CC(=O)c3cc(OC)ccc3O2)c1</smiles>

$6,3^{\prime}$ - dimetossiflavanone<smiles>COc1ccc2c(c1)C(=O)CC(c1ccccc1)O2</smiles>

6 - metossiflavanone<smiles>CC[N+](CC)(CC(=O)Nc1c(C)cccc1C)Cc1ccccc1</smiles>

benzoato di denatonio

Fig. 6 - Struttura di alcuni inibitori di specifici recettori del sapore amaro.

Nel 2014, sono stati individuate altre due sostanze in grado di comportarsi come inibitori del recettore T2R4 attivato dalla chinina: 
l'acido $\gamma$-amminobutirrico (GABA) e la $N \alpha, N \alpha$-bis(carbossimetil)-Llisina (BCML) (Fig. 7). Lo studio farmacologico ha permesso di stabilire che il GABA agisce come antagonista, mentre BCML si comporta come agonista inverso.<smiles>NCCCC(=O)O</smiles>

acido $\gamma$-amminobutirrico<smiles>NCCCC[C@H](C(=O)O)N(CC(=O)O)CC(=O)O</smiles>

$N \alpha, N \alpha$-bis(carbossimetil)-L-lisina

Fig. 7 - Altri inibitori dei recettori del sapore amaro.

Per l'uso pratico di questi bloccanti dell'amarezza, devono essere soddisfatti i seguenti requisiti:

i) i bloccanti devono essere efficaci a basse dosi. Un antagonista che deve essere usato in quantità equimolare o maggiore rispetto all'agonista non è efficiente;

ii) il composto deve funzionare quando applicato simultaneamente al composto amaro e deve possibilimente inibire tutti i recettori del sapore amaro;

iii) deve essere possibilmente di origine naturale ed essere disponibile in quantità sufficiente e a basso costo;

v) devono essere riconosciuti come GRAS (Generally Recognized as Safe) dalla Federation of Flavors and Extracts Manufacturers (FEMA).

\section{CONCLUSIONI}

Diverse sono le soluzioni a disposizione per coprire il sapore amaro dei farmaci, a seconda del tipo di specialità medicinale e della particolare applicazione.

I requisiti fondamentali richiesti perché l'uso in campo farmaceutico possono essere così riassunti:

a) richiedere il minor numero di passaggi di lavorazione;

b) poter essere condotto a temperatura ambiente, senza uso di apparecchiature specifiche;

c) fare uso del minor numero di eccipienti, che devono essere facil- 


\section{mente disponibili, a basso costo ed ammessi per l'uso in campo farmaceutico; \\ d) non devono avere effetti negativi sulla biodisponibilità del farmaco.}

\section{BIBLIOGRAFIA}

[1] Keast RSJ, Breslin PAS. Bitterness Suppression with Zinc Sulfate and NaCyclamate: A Model of Combined Peripheral and Central Neural Approaches to Flavor Modification, Pharmaceutical Research, 2005: 22: 19070-1977.

[2] Hussein MM, Bareclon SA. Taste-Masking Agents for Bitterness of Volatile Oils, US Patent 4,983,394, January 8, 1991.

[3] Yokoo T, Hirohata H. Composition for Oral Cavity, JP 05,000,931, January 8, 1993.

[4] Fuisz RC. Taste-Masking of Pharmaceutical Floss with Phenol, US Patent 5,028,632, July 2, 1991.

[5] Kurtz RJ, Fuller WD. Ingestibles Containing Substantially Tasteless Sweetness Inhibitors as Bitter Taste Reducers or Substantially Tasteless Bitter Inhibitors as Sweet Taste Reducers, US Patent 5,232,735, August 3, 1993.

[6] Stojanov M, Wimmer R, Larsen KIML. Study of the inclusion complexes formed between cetirizine and $\alpha$-, $\beta$-, and $\gamma$-cyclodextrin and evaluation on their taste-masking properties, J Pharm Sci., 2011:100:3177-85.

[7] Stojanov M, Larsen KL. Cetrizine release from cyclodextrin formulated compressed chewing gum. Drug Dev. Ind. Pharm., 2012:38: 1061-7.

[8] Patel AR, Vavia PR. Preparation and evaluation of taste masked famotidine formulation using drug/beta-cyclodextrin/polymer ternary complexation approach, AAPS Pharm Sci Technol., 2008;9:544-50.

[9] Gao R, Shao ZJ, Fan, ACL, Witchey-Lakshmanan LC, Stewart DC. Taste Masking of Oral Quinolone Liquid Preparations Using Ion Exchange Resins. US Patent 6, 514,492, February 4, 2003.

[10] a) Meyerhof W, Batram C, Kuhn C, Brockhoff A, Chudoba E, Bufe B, Appendino G, Behrens M. The molecular receptive ranges of human TAS2R bitter taste receptors, Chem. Sens. 2010:35: 157-170; b) Thalmann S, Behrens M, Meyerhof W. Major haplotypes of the human bitter taste receptor TAS2R41 encode functional receptors for chloramphenicol. Biochem. Biophys. Res. Commun. 2013: 435: 267-273.

[11] a) Slack JP, Brockhoff A, Batram C, Menzel S, Sonnabend C, Born S, Galindo MM, Koh S, Thalmann S, Ostopovici-Halip L, Simons CT, Ungureanu I, Duineveld K, Bologa CG, Behrens M, Furrer S, Oprea TI, Meyerhof W. Modulation of bitter taste perception by a small molecule hTAS2R antagonist, Curr. Biol. 2010:20:1104-1109; b) Roland WS, Gouka RJ, Gruppen H, Driesse M, van Buren L, Smit G, Vincken JP. 6-Methoxyflavanones as bitter taste receptor blockers for hTAS2R39, PLoS One, 2014: 9: e94451; c) Brockhoff A, Behrens M, Roudnitzky N, Appendino G, Avonto C, Meyerhof W. (2011) Receptor ago- 
nism and antagonism of dietary bitter compounds. J. Neurosci., 2011: 31 1477514782; d) Greene TA, Alarcon S, Thomas A, Berdougo E, Doranz BJ, Breslin PA, Rucker JB. Probenecid inhibits the human bitter taste receptor TAS2R16 and suppresses bitter perception of salicin, PLoS One, 2011: 6: e20123. 
